\title{
Intermédialités
}

Histoire et théorie des arts, des lettres et des techniques

Intermediality

History and Theory of the Arts, Literature and Technologies

\section{Conduite sociale et compétence écrite}

\section{Jack Goody}

Numéro 13, printemps 2009

Programmer

Programming

URI : https://id.erudit.org/iderudit/044047ar

DOI : https://doi.org/10.7202/044047ar

Aller au sommaire du numéro

Éditeur(s)

Centre de recherche sur l'intermédialité

ISSN

1705-8546 (imprimé)

1920-3136 (numérique)

Découvrir la revue

Citer cet article

Goody, J. (2009). Conduite sociale et compétence écrite. Intermédialités /

Intermediality, (13), 197-205. https://doi.org/10.7202/044047ar
Résumé de l'article

Dans cet essai, Goody poursuit ses réflexions sur la compétence écrite (literacy) dans le cadre d'une étude des relations entre la transmission de normes de conduite à l'intérieur des sociétés purement orales et leur communication par l'entremise de guides écrits. Il cherche ainsi à identifier les transformations sociales qu'entraîne la formalisation de contraintes de conduite par un code écrit afin de discuter des implications d'une différence dans le mode d'apprentissage et la médiation d'un discours de type juridique. En menant son investigation dans une perspective résolument comparatiste, Goody s'appuie sur ses recherches antérieures, qui englobe le langage des fleurs jusqu'aux us et coutumes de la société des LoDaaga, ainsi que sur différents phénomènes culturels, depuis les dix commandements jusqu'à l'expansion de l'État chinois. 


\title{
Conduite sociale et compétence écrite
}

\author{
JACK GOODY
}

12 rofesseur honoraire d'anthropologie sociale à l'Université de Cambridge, Jack Goody a profondément renouvelé avec ses recherches la vision que nous avions du rapport entre l'oralité et l'écriture. Depuis les années 1960, il s'est attaché à analyser de manière comparée la façon dont les changements dans les moyens de communication introduisent des transformations dans la nature des savoirs tout comme dans la distribution des pouvoirs, en particulier dans: Literacy in Traditional Societies (1968), La raison graphique (1978), La logique de l'écriture (1986), Entre l'oralité et l'écriture (1994), Pouvoirs et savoirs de l'écrit (2007). L'essai suivant, qui reprend la question d'une manière plus large à propos des guides de conduite, constitue une version augmentée de la conférence inaugurale «Behaviour and Literacy» donnée par l'auteur lors du colloque Behaviour Guides and Law. The Particular and the Universal of the (In)Formal, tenu les 3 et 4 décembre 2009 à l'Internationales Forschungszentrum Kulturwissenschaften à Vienne en Autriche ${ }^{1}$. Nous avons choisi de rendre le terme anglais literacy par l'expression «compétence écrite».

D'entrée de jeu, soulignons qu'il n'existe pas de société sans conduite de type juridique ${ }^{2}$. Nous utilisons ici le terme juridique comme un équivalent de ce qui est légal et de ce qu'est la loi dans les sociétés orales, lesquelles ne connaissent pas de cour de justice au sens où nous l'entendons. Tout le débat autour de la loi donne lieu à une série de confusions tant qu'on ne réalise pas qu'une conduite respectant la loi, soit une action qui s'accorde à des normes

1. Nous remercions les organisatrices du colloque, Karin Harrasser et Elisabeth Timm, d'avoir permis cette publication. La version originale de ce texte devrait paraître dans Behemoth-A Journal on Civilisation, vol. 3, 2010.

2. Traduction de l'anglais jural behaviour. 
sociales, prévaut déjà avant l'apparition de l'écriture et donc avant celle de la loi au sens formel du terme. L'écriture fut importante mais des conduites formelles, comme les coutumes par exemple, existaient bien avant celle-ci. Aucune société n’est anarchique ni dépourvue de critères de conduite, c'est-à-dire de normes de type juridique.

Si une «conduite conforme à la loi » et «des règles de bienséance » existent tout autant dans les sociétés purement orales que dans celles qui connaissent l'écriture, que change exactement ce que l'on nomme en anglais literacy ${ }^{3}$ ? D’abord, la compétence écrite augmente la portée de l'application d'une règle donnée puisqu'elle remplace la communication orale (qui se fait pour l'essentiel en personne) par une communication à distance par l'entremise du mot écrit. Pour cette raison, la portée de l'application des normes est beaucoup plus restreinte en l'absence de compétence écrite, alors que cette dernière permet aux codes de conduite de s'étendre à une communauté beaucoup plus grande. L'écriture peut favoriser une hégémonie et une domination tout autant qu'une «communauté ». Prenons l'exemple de la Chine. Lorsque l'État chinois étendit ses tentacules jusqu'au nord du Vietnam, une région qui parlait une tout autre langue, il diffusa en même temps l'écriture chinoise dans les territoires conquis ${ }^{4}$. Puisque ce système d'écriture n'est pas phonétique, il est susceptible de retranscrire n'importe quelle langue, aussi les caractères ont-ils été enseignés dans les écoles (tout comme au Japon ou en Corée aujourd'hui). En conséquence, les Vietnamiens ont été en mesure de lire les guides de conduite chinois, comme ceux indiquant comment traiter son père si l'on voulait faire preuve de "piété filiale».

On trouvait ainsi en Chine des conduites similaires - et nous pourrions en dire autant des rites funéraires et des mariages - qui se diffusaient de la Mandchourie, dans le nord du pays, jusque dans le Sud thaï, sans égard pour les différentes normes des communautés d'origine sous régime d'oralité. (Cette région immense forme aujourd'hui un seul marché et possède un langage écrit commun.) La notion chinoise de «croyance» dans les ancêtres, par exemple, tout comme le culte des morts, illustre une norme de conduite domestique qui s'est maintenue en usage grâce à sa formulation dans les textes confucéens. Ces textes écrits servaient d'instrument de domination coloniale, un peu comme

3. Nous choisissons de traduire literacy par «compétence écrite»; un néologisme correspondant à ce terme en français pourrait être «lettréité ».

4. Voir Jack Goody, Pouvoirs et savoirs de l'écrit, trad. de l'anglais par Claire Maniez, coordination par Jean-Marie Privat, Paris, La Dispute, 2007, p. 221 et sq. 
l'anglais ou le français l'ont été en Afrique et ailleurs. Ceux-ci appellent une conformité à la culture dominante. Et pas seulement dans ce cas.

Jusqu'à tout récemment, nous pouvions trouver dans les kiosques à journaux de la France rurale du Sud des guides épistolaires. Les variantes dialectales et régionales étaient expurgées de ces manuels - de fait, on pouvait être puni pour ne pas écrire en français du Nord. Chaque lettre devait commencer par une certaine phrase, dépendant du statut ou du rang de la personne concernée, et devait se terminer d'une manière prédéfinie: «Recevez... l'expression sincère de mes sentiments dévoués. » Cette phrase était la même pour tous, sans égard pour la spécificité des relations, aplanissant ainsi les différences locales.

Un autre exemple nous est donné par le grand nombre de livres et de guides sur le langage des fleurs qui apparurent en Europe au début du $19^{\mathrm{e}}$ siècle 5 . Selon certains de ses adeptes, ce langage tirait son origine d'un langage secret de l'Orient; pour d'autres, il renvoyait à la signification des fleurs dans la vie campagnarde ou dans la poésie - «mon amour est tel une rose rouge rouge». En réalité, même s'il a pu être lié à ces deux sources, il s'agissait d'un langage inventé dans lequel chaque fleur recevait une signification spécifique au sein d'une unique liste. Il était ainsi possible d'acheminer un message secret à l'être aimé comme : «rendez-vous dans le parc, au crépuscule ». Les différences locales ont, dans ce cas, également disparu. La liste rendait les choses étrangères à leur usage dans la vie ordinaire, où les roses pouvaient renvoyer non seulement à l'amour mais aussi au sang, ou même au comté de Lancashire - comme dans la guerre des Deux-Roses qui, en Angleterre, précéda l'ère des Tudor. Autrement dit, le sens ne dépendait pas d’une liste décontextualisée, comme dans un guide, il dépendait très directement du contexte, qu'il s'agisse d'amour ou de guerre, ou tout particulièrement de la région concernée: le sens attribué à une fleur différait donc d'un endroit à l'autre. À l'inverse, dans sa prétention à l'universalité, le texte ignorait ces différences et il offrait une seule interprétation pour toutes les circonstances. Et les listes avaient le même effet sur d'autres types de conduite. Dans l'exemple précédent, le soi-disant langage des fleurs était clairement lié à l'achat de fleurs chez un fleuriste et à leur composition en bouquet dans lequel chacune était porteuse d'une signification unique. Ces listes avaient un sens précis dans un contexte urbain spécifique mais non pas en tant que guide de coutumes rurales, car elles étaient l'invention d'une société orientée par le marché. Bien entendu, tous ces guides n’étaient pas identiques

5. Voir Jack Goody, La culture des fleurs, trad. de l'anglais par Pierre-Antoine Fabre, Éditions du Seuil, 1994, en particulier p. 267-295. 
mais ils avaient des points en commun, comme l'éducation de «l'inculte» ou l'apprentissage de la mobilité sociale dans une société où, plus que l'oralité, la compétence écrite était dominante. Ce cas précis nous fournit l'exemple d'une «tradition inventée » telle qu'exposée par des historiens comme Hobsbawn et d'autres: non la transcription d'une coutume issue d'une culture orale mais quelque chose qui fut «créé» dans une économie urbaine ${ }^{6}$. Le cas de la loi est très semblable.

Il est important de constater, et nos prédécesseurs des $19^{\mathrm{e}}$ et $20^{\mathrm{e}}$ siècles ne l'ont jamais fait, que le passage à la «civilité » (au sens restreint de «culture des cités») n’a pas seulement été un phénomène européen. Marx et beaucoup d'historiens pensaient le contraire. Norbert Elias a même écrit, en se basant sur une étude empirique de «livres d'étiquette» et de manuels de listes de bonnes manières, que c'est l'Europe qui avait, à la Renaissance, développé la «civilisation ». Ces guides n'étaient toutefois pas les premiers du genre, bien que leur diffusion ait été plus importante en raison de l'imprimerie. Comme on l'a déjà mentionné, on trouve un phénomène similaire en Chine avec les textes confucéens concernant la «conduite adéquate », par exemple quant aux relations avec le père, ou quant à l'ensemble des rapports prévalant lors de funérailles. La prescription de conduites similaires est commune dans d'autres sociétés marquées par l'écriture, mais elle l'est aussi dans les sociétés orales. Quand je vivais parmi les LoDagaa du nord du Ghana, tout le monde savait quelle conduite adopter, non seulement envers son père mais aussi envers chacun des autres membres de la communauté ${ }^{8}$. Par exemple, à la mort du frère de sa mère ou d'un oncle, on devait apporter un panier de graines pour préparer de la bière. Mais ces modes de conduite étaient très locaux, moins universels, et variaient selon les communautés.

Quelles différences l'apparition de l'écriture a-t-elle apportées à ce type d'activité ? J'ai soutenu qu'elle a rendu la réciprocité bien plus marquée. Il y a, par exemple, des cas où, dans une société orale, un homme ne peut apporter un panier de grains. Avec l'écriture, le don a pu être enregistré, un peu comme nous le faisons parfois avec les cartes de Noël. Celui qui n'en reçoit pas une année

6. Voir Eric Hobsbawm, «Inventer des traditions», dans Eric Hobsbawm et Terence Ranger (dir.), L'invention de la tradition [1983], trad. de l'anglais par Christine Vivier, Paris, Éditions Amsterdam, 2006, p. 11-25.

7. Voir Norbert Elias, La société de cour (1969), trad. de l'allemand par Pierre Kamnitzer et Jeanne Etoré, préface de Roger Chartier, Paris, Flammarion, coll. «Champs », 1985, en particulier le chapitre 3, p. 63-114.

8. Voir Jack Goody, Death, Property and the Ancestors: a Study of the Mortuary Customs of the LoDagaa of West Africa, Stanford, Stanford University Press, 1962. 
n'en enverra peut-être pas l'année suivante. Dans une société orale en revanche, on sait qu'il faut apporter un panier à son oncle, parce que c'est ainsi qu'on agit envers les oncles. Mais il est possible que l'on ait oublié si le panier a été ou non apporté; alors qu'avec l'écriture, on dispose d'un «aide-mémoire » permanent. Cela signifie que la réciprocité du don a pris avec l'écrit une forme différente, plus rigide.

Il semble qu'il en soit en général de même pour les guides de conduite. Ceux-ci ne sont pas transmis en personne comme dans les sociétés orales; ils existent en tant qu'objets tangibles pouvant être consultés en toutes circonstances et en dehors du contexte de l'action considérée. Ils deviennent plus abstraits, plus généraux et plus universels. Par exemple, au lieu d’impératifs tels que: «Tu ne tueras point de Français », on trouvera plutôt des exhortations générales comme: «Tu ne tueras point.» Or les sociétés humaines ne se comportent pas ainsi : elles tuent en telle circonstance et pas dans d'autres; elles tuent les roux mais non ceux qui ont les cheveux noirs, c'est selon. Avec l'écriture, on tend à présenter le problème en termes généraux, ce qui permet l'émergence de groupes oppositionnels de dissidents qui prennent le mot au pied de la lettre et refusent de prendre part à toute guerre. Comme dans le cas des Commandements, le mot écrit s’applique au monde en général. Dans les sociétés orales par contraste, les normes morales ne s'appliquent qu'à un contexte limité, de personne à personne, non à l'étendue d'une religion de l'écrit universaliste couvrant un large territoire. Un guide écrit devient ainsi plus répandu que celui qui ne l'est pas, mais il s'applique moins à des activités précises car tourné vers le général et plus universel, tel: «Tous les hommes sont frères. » En contrepartie, ces prescriptions sont plus théoriques, moins applicables et moins mises en pratique.

On a beaucoup débattu de la différence entre la loi formelle et les guides de conduite moins formalisés, bien qu'écrits. La principale différence tiendrait plutôt pour moi à la formalisation des contraintes de conduite par un code écrit appliqué par des tribunaux et des agents de l'ordre d'un côté, et des codes non écrits définissant la conduite adéquate de l'autre. Une différence réside également dans le contexte et dans les sanctions qui sont imposées à la suite d'un comportement interdit. Dans les temps anciens, les sanctions légales n'étaient pas jugées applicables à de nombreux différends domestiques ni aux conflits internationaux. Dans le premier cas, la famille «domestique » devait régler, jusqu'à récemment, ses problèmes de façon interne; dans le second, il n'existait pas, jusqu'à récemment également, de tribunal en position d'intervenir (faute d'État). Aujourd'hui, nous pouvons compter sur des institutions internationales, y compris des cours pénales (bien qu'elles soient peu adéquates). Ces 
deux situations ont donc en partie changé. Il existe, de plus, toute une part des relations interpersonnelles pour lesquelles une transgression du code n'apparaît pas assez importante pour que s'imposent des sanctions légales: ce sont des sanctions informelles qui entrent plutôt en jeu.

Dans ce cas, le développement de la loi n’a pas entravé celui des manuels de conduites. Ces derniers ont commencé à proliférer avec l'apparition de l'imprimerie, comme dans le cas des livres de recettes ou de ceux qui révélaient le langage des fleurs. Ce ne fut toutefois pas là le facteur le plus décisif. Certes, ils se sont multipliés lors de la Renaissance italienne, mais croire que ces livres ou les comportements concernés sont apparus à cette époque (comme le fait Elias dans sa célèbre étude), c'est mal comprendre la nature de la communication humaine, et plus précisément les effets de la compétence écrite à travers le monde. Bien entendu, leur importance s'est accrue en Europe à cette époque, à un moment où il existait davantage de mouvement entre les groupes sociaux, de sorte que les processus normaux d'inculcation des conduites «raffinées» par l'apprentissage domestique ne pouvaient pas fonctionner: on apprenait davantage dans les livres que dans la famille - par l'éducation plutôt que par la socialisation. Par ailleurs, l'effet de généralisation des guides de conduite n’a pas seulement une dimension spatiale, il a aussi une dimension temporelle: lorsque les mœurs et les coutumes changent, ces guides demeurent inchangés, propres à une époque antérieure, comme dans le cas des tabous juifs issus du Lévitique, ou de l'interdit de consommation du porc dans le monde juif et musulman. Il arrive que les codes de conduite ne soient pas écrits; toutefois, quand ils le sont, ils résistent davantage au changement et aux transformations. Ce genre de guide se montre particulièrement utile lorsque change le contexte dans lequel vivent les gens, comme ce fut le cas lors de l'essor de la bourgeoisie. L'importance que prit la mobilité sociale fit en sorte qu'on ne savait plus quels vêtements porter (le vêtement selon le rang, comme dans la loi somptuaire, avait perdu de sa pertinence), quelle nourriture servir, comment s'adresser à ses supérieurs: tous ces éléments exigeaient des guides de conduite, non parce que la structure sociale était statique mais parce qu'un certain mouvement était sensible en son sein. De même, la «mode» s'est mise à jouer un rôle plus important dans la société dans son ensemble. En fait, certains cercles sociaux se sont maintenus fermés par de fréquents changements de mode, comme ce fut le cas d'une manière formelle lors de l'établissement de la cour de Louis XIV et des soieries de Lyon. Pour être à la mode, il fallait non seulement être bien vêtu d'une manière traditionnelle, il fallait encore l'être d'une manière contemporaine qui tenait compte de changements de plus en plus réguliers (tous les six mois). 
Cependant, l'Europe n'a aucunement été la première à s'engager dans la mode. Le changement a toujours existé, même dans les sociétés et civilisations qualifiées de traditionnelles. Dans celles-ci, toutefois, le changement se produisait à un autre rythme, et il était plus difficile de le remarquer. Ce rythme s'est accru avec l'apparition de l'écriture, puis davantage avec celle de l'imprimerie puis de l'ordinateur. Ce dernier a d'ailleurs d'une certaine façon remplacé le guide écrit. On peut consulter le Web pour s'instruire de la conduite adéquate ou trouver les publicités indiquant la robe appropriée.

Les guides de conduite écrits concernent habituellement les formes d'action les moins importantes: livres de recettes, répertoires du langage des fleurs, comment s'adresser à quelqu'un, en personne ou par lettre, ce qui implique souvent des listes sur lesquelles je reviendrai. Les dix commandements des chrétiens (mais ceux d'ailleurs aussi) impliquent des contraintes et des impératifs d'une importance supérieure - «Tes père et mère honoreras » - et composent ainsi une sorte de code de conduite; ils sont parfois intégrés dans un code juridique: «Tu ne voleras pas. » Ce que nous avons qualifié de «moins important» n'est pas toujours considéré comme tel par les acteurs. Le fait d'offrir des chrysanthèmes à une épouse catholique brise un important tabou qui n'existe pas dans les pays protestants. Ces formes de conduite sont souvent stratifiées par classe (manger des pois à la fourchette, par exemple) ou par groupe ethnique (ne manger qu'avec une fourchette, comme aux États-Unis) ; les Commandements, en revanche, reposent sur une autorité religieuse, aussi ont-ils une importance supérieure (comme dans le cas du vol ou de l'adultère).

Guides et commandements peuvent tous deux s'établir sous forme de « liste». Cette mise en liste caractérise, comme j’ai pu le soutenir, les sociétés de l'écrit. Des listes apparaissent souvent dans les premières tablettes d'argile de Mésopotamie où, par exemple, des listes d'arbres étaient utilisées pour l'enseignement de la lecture (et de l'écriture) dans les écoles établies à l'époque?. Ces listes prirent ensuite un caractère normatif. De manière analogue, les guides de conduite détaillaient par des listes les actions convenables; les préceptes y étaient toutefois empruntés à l'éducation orale puis traités d'une manière abstraite. Autrement dit, l'écriture tire la conduite hors du contexte singulier de l'action sociale

9. À propos des listes, voir Jack Goody, La raison graphique: la domestication de la pensée sauvage, trad. de l'anglais et présenté par Jean Bazin et Alban Bensa, Paris, Les Éditions de Minuit, 1979, p. 140-196; sur le développement de l'écriture en Mésopotamie: Entre l'oralité et l'écriture, trad. de l'anglais par Denise Paulme et révisé par Pascal Ferroli, Paris, Les Presses universitaires de France, coll. «Ethnologies», 1994, p. 47 et sq. 
et lui donne un traitement plus général. Les listes d’arbres ne fonctionnent pas autrement. Je soutiens qu'il n'existait à peu près aucune occasion d'établir des listes d'arbres dans les cultures orales: on y observait chaque arbre séparément, quand on le faisait, dans son contexte réel - et ainsi, le général y était implicite. De même pour les guides et les commandements : on ne produisait de listes de ceux-ci que de manière décontextualisée, dans un contexte qui impliquait, lui, l'écriture.

L'écriture permet de communiquer avec un auditoire plus étendu, mais moins personnalisé. Un plus grand nombre de personnes adoptent le même code écrit, ce qui élimine ou limite la diversité locale. L'imprimerie ne fait que renforcer ces tendances, puisqu'elle produit d'un seul coup un grand nombre de copies similaires - une situation très différente de celle caractérisant les cultures manuscrites. Il en résulte une baisse de la diversité locale et un accroissement de l'uniformité. Ainsi les règles sont mieux définies (avec moins d'exceptions) lorsqu'elles sont écrites. Elles font davantage autorité, sont plus précises, puisqu'un grand nombre de personnes peut agir selon un même texte écrit, et plus difficiles à ignorer, particulièrement dans un contexte religieux - comme dans le cas des Commandements qui ne constituent pas seulement une loi morale mais la loi divine.

En résumé, les guides de conduite n’ont en eux-mêmes rien de nouveau. Dans les cultures orales, les gens apprenaient comment se comporter dans un contexte familial ou villageois. L'éducation ne se faisait que rarement de manière délibérée. Dans la société LoDagaa du Bagré - dont j’ai recueilli les mythes sur une période de quarante $a n s^{10}-$, on révèle expressément aux néophytes, ceux qui sont en voie d'être initiés comme membres, ce qu'implique cette appartenance: par exemple le type de nourriture à consommer et le moment de le faire, quels objets employer, etc. Il y a des parties du Bagré qui visent spécifiquement l'apprentissage de manières et de comportements que seuls les initiés connaissent.

Quels sont finalement les effets des guides de conduite, quels sont ceux produits par la compétence écrite?

1) Extension: La compétence écrite diffuse des formes de conduite sur une vaste échelle, dans l'espace et dans le temps, et ce, d'autant plus qu'elle est associée à une doctrine religieuse - comme les tabous alimentaires de l'Exode.

10. Voir Jack Goody, The Myth of the Bagre, Oxford, Clarendon Press, [Londres, Oxford University Press], 1972. 
2) Mobilité sociale: Non seulement la compétence écrite généralise en ce sens, mais elle constitue aussi un mécanisme d'apprentissage décontextualisé; une éducation basée sur la lecture de ce qu'il faut faire s'oppose à un apprentissage par la socialisation, de personne à personne. Aussi instruit-elle les gens des classes inférieures de la manière d'agir pour devenir membre des classes supérieures.

3) Formalisation: La compétence écrite semble durcir et rigidifier le code de comportements issu du monde oral; une fois celui-ci écrit, il est plus difficile d'y échapper.

4) Universalisation: La compétence écrite, et c'est peut-être là un aspect du premier point, aplanit les différences de contexte et fournit une même norme pour tous.

Nous avons abordé cette problématique comme étant une question de l'évolution culturelle entre les sociétés de l'écrit (dotées de codes juridiques, de tribunaux et d'agents de l'ordre, comme le dit Malinowski ${ }^{11}$ ) et celles d'une pure oralité. Enfin, bien que l'informalité des sociétés de l'écrit diffère de la pratique des sociétés orales, il n'en existe pas moins une certaine relation entre les deux.

(Traduit de l'anglais par Patrick Poulin)

11. Voir Bronislaw Malinowski, Mours et coutumes des Mélanésiens (Trois essais sur la vie sociale des primitifs) [1933], trad. S. Jankélévitch, Paris, Payot, 1975, p. 52 et sq. 